\title{
Funktionen von Interregionalismus - Eine globale Perspektive
}

\section{Georg Lammich}

Online publiziert: 20. Februar 2020

(C) Der/die Autor(en) 2020

\section{Einleitung}

Trotz einer langen Tradition von Group-to-group-Dialogen, insbesondere im Rahmen der Außenbeziehungen der Europäischen Union, ist Interregionalismus ein relativ junges Phänomen, dessen Genese häufig in einen Kontext mit Globalisierung, Regionalismus und dem Aufstieg der Triade-Regionen (EU, USA, Ostasien) gestellt wird. (Roloff 2006; Rüland 2010; Baert et al. 2014; Doidge 2011).

Interregionalismus versucht dabei einen Erklärungsansatz für die, besonders nach Ende des Kalten Krieges, entstandenen Netzwerke und Beziehungen zwischen oder mit Beteiligung unterschiedlicher regionaler Organisationen zu liefern. (Hänggi et al. 2006).

Viele der Untersuchungen zum Interregionalismus haben sich auf inter- und transregionale Foren mit Beteiligung der Europäischen Union konzentriert, mit einem besonderen Fokus auf die EU-ASEAN-Beziehungen (Gilson 2005; Yi 2012; Allison 2015;) oder die transatlantische Kooperation (Aggarwal und Fogarty 2005; Santander 2010; Mattheis und Godsäter 2018). Seit den siebziger und achtziger Jahren, als „Interregionalismus“ meistens synonym zu den Gruppendialogen der EU genutzt wurde, ist sowohl die empirische Vielfalt als auch die konzeptionelle Komplexität von interregionaler Kooperation gestiegen und eine Reihe von unterschiedlichen empirischen Phänomenen wird inzwischen unter dem Begriff zusammengefasst (Baert et al. 2014, S. 174; Drechsel 2016, S. 23).

Dr. G. Lammich $(\bowtie)$

Institut für Politikwissenschaft, Universität Duisburg-Essen, Lotharstraße 65, 47057 Duisburg, Deutschland

E-Mail: Georg.Lammich@uni-due.de 


\section{Die Außenbeziehungen regionaler Organisationen}

Das Konzept einer regionalen Agency wurde erstmals im Zusammenhang mit dem Transformationsprozess der Europäischen Union - von einem Instrument wirtschaftlicher Kooperation zu einem politischen Akteur, der gezielt versucht, externe Bedingungen zu beeinflussen - diskutiert (Hettne 2008, 2014). Als Erklärungsansatz für die Übernahme klassischer nationalstaatlicher Aufgaben durch regionale Akteure wird dabei besonders die Komplexität der Herausforderungen der Globalisierung aufgeführt, die von Nationalstaaten nicht mehr allein bewältigt werden können: ,[...] through the pooling of resources and sovereignty, states hope to contain the disruptive and disquieting forces of globalization, to increase their bargaining power in global multilateral forums [...] and to curtail the growing influence of transnational actors " (Hänggi et al. 2006, S. 6). Die wachsende Zahl transnationaler Interdependenzen erodiert die klassische Kongruenz zwischen sozioökonomischen Handlungen und politischen Grenzen und erfordert regulative Eingriffe, die die Reichweite eines einzelnen Staates übersteigen. Aber auch neue Arten von Konflikten, der Klimawandel, Finanzkrisen etc. bieten einen Anreiz für Staaten, ihre Ressourcen zu bündeln und gemeinsam auf externe oder transnationale Bedrohungen zu reagieren. (Rüland 2006; Doidge 2011; Carrapatoso 2011) Mit der Verdichtung regionaler Kooperation, der Etablierung regionaler Mechanismen und Institutionen sowie einer teilweisen Kompetenzabgabe von der nationalen auf die regionale Ebene steigt die Handlungskapazität regionaler Akteure, woraus in vielen Fällen auch die Aufnahme eigener Außenbeziehungen resultiert (Hänggi et al. 2006, S. 5-7). Regionen und ihre institutionalisierten Vertretungen sind im Falle einer Externalisierung ihrer Beziehungen nicht mehr nur die Arenen für die Interaktion staatlicher Akteure, sondern eigenständig handelnde Subjekte im Kontext internationaler Beziehungen. „Regional actorship is used as a summary concept for a region's ability to influence the external world and for instance engage in interregionalism " (Hettne 2014, S. 57).

Den Grad organisatorische Charakteristan „actorness“, der aus einer solchen theoretischen Perspektive für die Aufnahme eigenständiger Außenbeziehungen erforderlich ist, haben inzwischen auch Regionale Organisationen außerhalb Europas erreicht. Sie haben eine eigene Handlungsfähigkeit entwickelt, die es ermöglicht, kohärente Strategien zu formulieren und diese zumindest graduell in einen Dialog mit externen Partnern einzubringen (Hulse 2014, S. 562). Folgt man der existierenden Literatur zu regionalen Akteurskapazitäten (Bretherton und Vogler 2006, Acharya und Johnston 2007; Hettne 2014), führt diese institutionelle Evolution auch bei nichteuropäischen ROs zu einem gestärkten Bewusstsein, als eigenständige Akteure im internationalen System agieren zu können. Dieses Selbstverständnis der eigenen Handlungsfähigkeit kann sich in den Versuch übersetzen, autonome Beziehungen $\mathrm{zu}$ anderen Regionen und Staaten aufzubauen und eigene außenpolitische Ziele zu artikulieren (Engel 2018, S. 30). Bei ihrem Werdegang zu globalen Akteuren gestalten diese Regionalen Organisationen aktiv das internationale politische System mit, von dem sie gleichzeitig selbst ein Teil sind. (Väyrynen 2003, S. 43). Hänggi et al (2006) zeigen durch Untersuchungen der EU Außenbeziehungen mit ASEAN, MERCOSUR und dem südlichen Afrika sowie der Analyse peripherer Arrangements wie dem Forum for East Asia-Latin America Cooperation (FEALAC), dass sich in 
den letzten zwei Jahrzehnten Regionen durch die Aufnahme von externen Beziehungen zu anderen Regionen oder Staaten zunehmend als eigenständige Akteure in den Internationalen Beziehungen (IB) etablieren. Hänggi et al. berücksichtigen dabei besonders die Beziehungen innerhalb der Triade und transregionale Kooperationsformen wie die Asia Pacific Economic Cooperation (APEC) und das Asia-Europe Meeting (ASEM), denen sie eine eigenständige internationale Handlungsfähigkeit zuschreiben (Hänggi et al. 2006, S. 2). Bei der Diskussion um die internationalen Akteurskapazitäten Regionaler Organisationen - und damit einhergehend um Interregionalismus - stehen besonders die Außenbeziehungen der Europäischen Union im Fokus. Unter den Schlagwörtern „Transregionalismus“ und „Interregionalismus“ gibt es eine steigende Zahl von Untersuchungen, die sich mit der Analyse regionaler Außenbeziehungen beschäftigen. ${ }^{1}$ Die Begriffe ,Transregionalismus“ und „Interregionalismus“"werden dabei mangels einer klaren Definition für das Phänomen von Regionen als außenpolitischen Akteuren häufig austauschbar eingesetzt (vgl. Aggarwal 1998; Rüland 1999). Laut Hettne handelt es sich bei Transregionalismus um , institutions and organization mediating between regions “ (Hettne 2004, S. 11), während Interregionalismus den Zustand nach der institutionellen Formalisierung dieser Beziehung beschreibt. Söderbaum und Stalgren (2010) sieht Transregionalismus als Erweiterung des eng definierten Region-to-region-Prozesses innerhalb eines formellen, meistens intergouvernementalen Rahmens. Transregionalismus ist noch offener konzipiert als Interregionalismus und hebt ab auf Beziehungen zwischen Regionen mit schwach ausgeprägten Akteursqualitäten (Söderbaum und Stalgren 2010, S. 6). Neben einer größeren Heterogenität und diffuseren Mitgliedschaft der beteiligten Akteure können auch Staaten und andere Akteursgruppen (Institutionen, Bewegungen etc.) maßgeblich am transregionalen Kooperationsprozess beteiligt sein (Tsardanidis 2010, S. 219). Aggarwal und Fogarty definieren Interregionalismus als „[...] the pursuit of formalized intergovernmental relations with respect to commercial relationships across distinct regions “ (Aggarwal und Fogarty 2004, S. 1). Während bei dieser Sichtweise vor allem ökonomische Gesichtspunkte im Vordergrund stehen, bietet Roloff einen guten Ausgangspunkt, um die Komplexität von Interregionalismus in ihrer Gesamtheit zu erfassen. „Interregionalismus beschreibt die politisch gewollte und daher aktiv betriebene Verdichtung der interregionalen Aktivitäten “ (Roloff 2001, S. 99). Interregionalismus wird nicht als rein ökonomisches, sondern als multidimensionales Phänomen betrachtet, das in einem post-hegemonialen internationalen System auftritt. Anders als der Regionalismus zu Zeiten des Kalten Krieges, der von einer US-amerikanischen liberalen Hegemonie und der „Open-Regionalism-Doktrin“ geprägt wurde, bildet der heutige Interregionalismus ein innovatives Paradigma internationaler Beziehungen, das Teil einer zunehmenden vertikalen Differenzierung internationaler Institutionen ist, die vom globalen bis zum lokalen Level reicht (Rüland 2010, S. 157). Interregionale Beziehungen sind damit nicht nur Bausteine für ein liberalisiertes globales Handelssystem, sondern bilden eine eigene Bezugsebene für die externen Beziehungen von ROs, die zwar mit dem

\footnotetext{
1 Alternative Konzepte für solche Organisationsformen wären der „Mega-Regionalism“ (Yamamoto 1996), „Transcontinentalism“ (Roloff 2001) und „Pan-Regionalism“ (Gilson 2002).
} 
globalen System interagieren können, aber nicht zwangsläufig eine Integration in dieses anstreben.

\section{Theoretische Konzeptualisierung von Interregionalismus}

Die verschiedenen multidimensionalen Ausprägungen von Interregionalismus und der vergleichsweise kurze Zeitraum der wissenschaftlichen Auseinandersetzung mit den Außenbeziehungen Regionaler Organisationen - besonders außerhalb der Europäischen Union - führen zu einer Vielfalt teils sehr unterschiedlicher Konzeptualisierungen des Begriffes. Schon bei der Definition des Untersuchungsobjektes gibt es große Unklarheiten; so wird beispielsweise das Forum der Asia-Pacific Economic Cooperation (APEC) je nach Blickwinkel als ,multilateraler Interregionalismus“ (Faust 2006), „Mega-Region“ (Hänggi et al. 2006), ,transregionales Forum“ (Aggarwal 1998), ,pan-regionales Arrangement“ (Gilson 2002) oder als „Region“ (Ravenhill 2001) definiert.

$\mathrm{Zu}$ Zeiten des Kalten Krieges gab es, bedingt durch die systemische Bipolarität und den damit einhergehenden Fokus auf transregionale Allianzen, wenig Spielraum für interregionale Beziehungen; dementsprechend wurde dieses Forschungsfeld in den IB, mit Ausnahme einzelner Ansätze wie dem ,extra-regional echoing“ (Avery 1973, S. 550), auch kaum wahrgenommen. Zwar gab es schon seit den 70er Jahren Beispiele interregionaler Dialoge wie den EU-ASEAN-Gipfel, aber die theoretische Auseinandersetzung mit dem Phänomen der Außenbeziehungen Regionaler Organisationen blieb weitestgehend aus. Eine Ausnahme bilden Kaisers Studien (1968, 1981) über die Interaktionen regionaler Subsysteme. Kaisers Beobachtungen zu der Interaktion zwischen der NATO und den Teilnehmerstaaten des Warschauer Vertrages, oder auch zwischen der Latin American Free Trade Association (LAFTA) und der EWG, und der Versuch der Typologisierung unterschiedlicher Kooperationsformen bilden einen wichtigen Baustein für die spätere Auseinandersetzung mit Interregionalismus. „Attempts at building theory on regional processes (in particular on integration) and on international politics in a wider sense should draw upon one another more intensively_indeed, should explicitly proceed jointly." (Kaiser 1968, S. 107). Während Kaiser schon früh versucht, interregionale Phänomene auch theoretisch zu erfassen, blieb diese Debatte aufgrund der geringen Fallzahl interregionaler Arrangements und deren Beschränkung auf sogenannte „low politics“ (Gardini und Malamud 2014, S. 3) eine Randerscheinung der IB. Erst seit dem Ende des Kalten Krieges gibt es wieder mehr Bewegung im Bereich der regionalen und interregionalen Beziehungen - und neue Arrangements jenseits der EU-dominierten Group-to-group-Dialoge sind entstanden. Insgesamt sind über 100 der 254 bei der WTO registrierten RTAs (Regional Trade Agreements) in den letzten zwei Dekaden zustande gekommen und auch die Zahl der Regionalen Organisationen hat sich in allen Weltregionen vervielfacht (Panke 2019, S. 3). Obwohl die EU immer noch ein Schwergewicht im expandierenden Beziehungsnetzwerk zwischen regionalen Gruppen ist, können sich auch zunehmend interregionale Beziehungen ohne Beteiligung Europas etablieren. Dabei sind neue, häufig vielschichtige Formen interregionaler Beziehungen mit einer großen Varianz bei der institutionellen Ausgestaltung entstan- 
den (van der Vleuten und Ribeiro-Hoffmann 2013). Aus einer theoretischen Perspektive orientieren sich die meisten Untersuchungen an den aktuellen Diskursen in den Internationalen Beziehungen und dem Versuch, das Realismus-Liberalismus-Paradigma zu überwinden (Hänggi et al. 2006, S. 6). „Generally, the existing literature demonstrates a popular trend of combining a few selected theories, especially neorealism, liberal-institutionalism and social constructivism, to explain inter-regional interactions [...]“" (Yi 2012, S. 38). Aus der Perspektive des Realismus versuchen Regionale Organisationen bzw. ihre Mitgliedsstaaten, im Kontext eines anarchischen internationalen Systems Koalitionen zu bilden, um sich gegen externe Bedrohungen zu schützen oder in internationalen Regimen und Institutionen Ressourcen zu mobilisieren, um die eigenen Ziele durchzusetzen (Cammack 1999, S. 14; Rüland 2010; Doidge 2011). Das Handeln folgt dabei einer angenommenen Konkurrenzsituation, bei der der wirtschaftliche oder politische Erfolg oder Gewinn eines Akteurs einem Misserfolg oder Verlust eines anderen gegenübersteht. Akteure nutzen Regionalismus und in logischer Konsequenz auch Interregionalismus als eine defensive Strategie, um im globalen Wettbewerb um institutionelle Macht und Einfluss zu bestehen (Faust 2004, S. 749). Der rationale agierende und somit Nutzen maximierende Staat ist hier der entscheidende Akteur, der interregionale Institutionen für die Durchsetzung seiner Ziele instrumentalisiert. Kooperationsentscheidungen werden durch nationale Interessen bestimmt und resultieren (wenn überhaupt) nur in kurzfristigen Arrangements, die auf die relativen Gewinne der beteiligten Staaten ausgerichtet sind.

Im Gegensatz dazu betont der neoliberale Institutionalismus nicht die Hemmnisse, sondern die Chancen von Kooperation im internationalen System und die Möglichkeit kollektiver Gewinne. Aus einer institutionalistischen Perspektive begründet sich das Kooperationsinteresse der Akteure durch die zu erwartenden langfristigen Sicherheits- und Wohlstandseffekte interregionaler Kooperation, wobei im Falle einer angestrebten Wohlstandssicherung eher kooperative Impulse ausschlaggebend sind, während bei einem Wohlstandsgewinn kompetitive Motive im Vordergrund stehen (Maull und Okfen 2006, S. 218). Interregionalismus bietet den Akteuren eine Möglichkeit, komplexe Interdependenzen zu managen, Vertrauen aufzubauen und Unsicherheiten zum gegenseitigen Nutzen der beteiligten Partner zu reduzieren (Hulse 2016, S. 35). Interregionalismus hat damit das Potenzial, eine neue Ebene in der internationalen Arena zwischen der multilateralen und der regionalen Ebene $\mathrm{zu}$ besetzen, wenn die Akteure ihre gemeinsamen Interessen und Ziele erkennen und abstimmen (Hwee 2008, S. 10; Yi 2012, S. 34): ,[...] interregionalism can address problems of multilateral cooperation amongst a large number of state actors [...]" (Hulse 2016, S. 38). Bedingt durch die hohen Kosten für die Einrichtung einer solchen interregionalen Bezugsebene und die negativen Effekte im Falle eines Wegfalls dieser Ebene werden diese Institutionen im Gegensatz zur realistischen Sichtweise als beständig angesehen. Solch eine Institution hat damit das Potenzial, sich zu einem eigenständigen Akteur in der internationalen Arena zu entwickeln. Sie bietet ihren Mitgliedern Informationen und Raum für reziproke Handlungen und ggf. Sanktionsmechanismen. Einmal etabliert, bietet eine solche Institution nicht nur einen Kooperationsrahmen, sondern hat auch Einfluss auf die Erwartungen der beteiligten Staaten an den Nutzen von solchen Arrangements (Yi 2012, S. 34). Staaten 
sind zwar auch hier die entscheidenden Akteure; sie werden aber differenzierter betrachtet als im Realismus. So können auch andere nichtstaatliche Interessengruppen auf verschiedenen Sub-Ebenen durch Mobilisierung und Lobbyarbeit Einfluss auf interregionale Prozesse nehmen (vgl. Costa und Dri 2014).

Autoren wie Maull und Okfen (2006) oder Roloff (2001) betrachten interregionale Dialoge als das Ergebnis eines Prozesses, der neorealistische und institutionalistische Annahmen miteinander vereint, womit sowohl Machtstreben als auch kooperationsorientierte Motive interregionaler Zusammenarbeit begründet werden. „The variations of this mix are dependent on context as well as cognitive factors, i.e. how previous interactions and historical experiences and the resultant role expectations have shaped actor perceptions. " (Hänggi et al. 2006, S. 10). Andere Forscher wie Richards und Kirkpatrick (1999) versuchen durch eine Kombination von neoliberalen und realistischen Ansätzen eine Machtverschiebung - weg von staatlichen Akteuren, hin zu regionalen und interregionalen Strukturen - aufzuzeigen. ,We suggest that changes in the structure of production, trade and finance are impinging on the nature and location of power, that it is increasingly difficult for ,national 'objectives to be pursued and managed through national strategies, and that this accounts for the emergence of new regional institutions and interregional arrangements to build on existing bilateral relations and reinforce multilateral initiatives" (Richards und Kirkpatrick 1999, S. 685).

Neben Realismus und Liberalismus bzw. einer Kombination dieser Ansätze bietet der Konstruktivismus eine weitere Perspektive für die Erklärung interregionaler Kooperation (Baert et al. 2014, S. 171). Konstruktivismus inkludiert bei der Analyse regionaler und interregionaler Kooperation zwar auch die materielle Ebene; es werden aber zusätzlich ideelle Faktoren als mögliche erklärende Variablen für die Ausprägung von Kooperation herangezogen. „Although capable of providing coherent explanations, materialist arguments often present some limitations in their analysis of regionalism due to their emphasis on material dimensions, namely, power and benefit maximisation. Constructivism, however, pays special attention to ideational 'dimensions" (Prieto 2003, S. 2). Diese Ansätze beleuchten vor allem den Einfluss von Identität, Normen und Werten der beteiligten Akteure für den Verlauf interregionaler Kooperation, aber auch die Effekte von interregionaler Interaktion auf ebendiese ideellen Faktoren. Regionale und staatliche Akteure versuchen durch externe Beziehungen, regionale kollektive Identitäten zu bilden oder zu stärken (Maull und Okfen 2006, S. 218). Die Annahme des Realismus, dass Staaten in einem anarchischen System agieren, wird zwar nicht negiert; aber nicht Macht, sondern normative Strukturen sind entscheidend für das Verständnis des Akteursverhaltens (vgl. Wendt 1994). Bedingt durch ein Informationsdefizit und die Möglichkeit irrationalen Handelns greifen Staaten auf kognitive Verhaltensmuster - geprägt durch Erfahrungen und Ideen - zurück, um die Kosten und Nutzen interregionaler Kooperation abzuwägen (Yi 2012, S. 37). Die Bereitschaft zu Engagement in interregionalen Arrangements ist Teil eines sozialen Lernprozesses, der die Identität und die Interessen der Akteure und damit ihre Haltung zu interregionaler Kooperation verändert.

Während die meisten Untersuchungen zum Interregionalismus die materielle (Realismus), institutionelle (Liberalismus) oder ideelle (Konstruktivismus) Perspektive bzw. eine Kombination aus diesen einnehmen, gibt es vereinzelt Kritik an 
der Anwendbarkeit dieser theoretischen Ansätze. „Inter-regionalism has to be seen as a distinctive form of international diplomacy, as a category of its own. As such, tackling it from theoretical perspectives may require rethinking the outcome-oriented theories of international co-operation, be they rationalist or constructivist" (Maull und Okfen 2003, S. 248).

Während Maull und Okfen keine theoretische Alternative zur Untersuchung interregionaler Interaktionen anbieten, versucht Farrell (2004) sich dem Phänomen des Interregionalismus aus einer akteurstheoretischen Perspektive zu nähern und bringt eine Abwandlung von Pedersens „Cooperative Hegemony Approach“ ins Spiel. „The cooperative hegemony approach involves the use of soft power through engagement in cooperative arrangements linked to a long-term strategy" (Farrell 2004, S. 7). Während die Annahme eines kooperativen Hegemonen sicherlich einige Aussagekraft für interregionale Arrangements mit Beteiligung der EU (wie etwa die EUASEAN- oder die EU-ACP-Partnerschaft) hat, werden andere Formen interregionaler Kooperation ohne Beteiligung der EU nicht berücksichtigt.

Auch bei der Klassifizierung erschwert die Vielzahl der Ausprägungen von Interregionalismus eine klare Unterteilung und Definition der unterschiedlichen Formen dieses Phänomens. Seit den siebziger und achtziger Jahren, als „Interregionalismus“ meistens synonym zu den Gruppendialogen der EU genutzt wurde, ist sowohl die empirische Vielfalt als auch die konzeptionelle Komplexität von interregionaler Kooperation gestiegen und eine Reihe von unterschiedlichen empirischen Phänomenen wird inzwischen unter dem Begriff zusammengefasst (Drechsel 2016, S. 23).

Hänggi definiert schon in einer seiner frühen Arbeiten über Interregionalismus die verschiedenen Arten von externen Beziehungen, die eine Region haben kann, je nach Art des Partners, mit dem die Beziehung unterhalten wird (Hänggi 2000). Die typologische Ausdifferenzierung des Begriffes von Hänggi $(2000,2006)$ in reinen, transregionalen und hybriden Interregionalismus bietet wohl die etablierteste Kategorisierung von Interregionalismus. Interregionalismus beschreibt dabei immer einen Handlungszusammenhang, der sich von der regionalen Regelungsstruktur abgrenzt und über eigene institutionelle und organisatorische Charakteristika verfügt (Drechsel 2016, S. 29).

\section{Die Funktionslogik von Interregionalismus}

Internationale Beziehungen werden zunehmend nicht nur als ausschließlich von machtpolitischen bzw. kooperationsorientierten Motiven bestimmte Prozesse angesehen, sondern als eine komplexe Mischung aus Policies, die sowohl von neorealistischen wie auch von institutionalistischen Prinzipien beeinflusst werden. Die Variationen dieser Mischung sind abhängig vom Kontext und von kognitiven Faktoren, d.h. davon, wie vorangegangene Interaktionen und historische Erfahrungen die Sichtweise der Akteure beeinflusst haben (Hänggi et al. 2006, S. 10). Roloff kombiniert in seiner Untersuchung über interregionale Beziehungen (2001) den neorealistischen Ansatz von Waltz (1979) mit der Interdependenztheorie von Keohane und Nye (1977) und ist somit in der Lage, aufzuzeigen, dass interregionale Beziehungen sowohl ein Resultat von kooperativem Verhalten von Akteuren als auch von (institu- 
tionellem) Balancing sind. Das Zusammenspiel von Interdependenz und Polarisierung schafft dabei Raum für die Entstehung flexibler interregionaler Kooperationsstrukturen, die unterschiedliche Funktionen ausüben. Diese besonders von Rüland (2001, 2002a, 2010) und Doidge (2011) entwickelten Funktionen interregionaler Arrangements orientieren sich an Großtheorien der Internationalen Beziehungen (Neo-)Realismus, (liberalem) Institutionalismus und Konstruktivismus - und bieten einen Rahmen für die Einschätzung der Leistungsfähigkeit interregionaler Prozesse und der Interessen, die teilnehmende Akteure damit verknüpfen (Baert et al. 2014). Rüland (2001, 2002a) benennt dabei fünf Funktionen von Interregionalismus, Balancing, Institution Building, Rationalizing, Agenda Setting, und Identity Building. Rüland (2002a) befand diese Funktionen von Interregionalismus zwar für theoretisch begründet, aber nicht als empirisch überprüft: „As stated earlier, most of the functions attached to inter- and transregional fora rest less on empirical evidence than on theoretical deduction " (Rüland 2002a, S. 7). Seitdem haben aber verschiedene Studien Belege für diese Funktionen anführen können (Valle 2008; Hardacre und Smith 2009; Hardacre 2009) und gezeigt, dass sie - wenn auch in stark variierenden Intensitäten - in unterschiedlichen interregionalen Kontexten ausgeübt werden.

Balancing wird von Rüland (2001) als eine der wichtigsten externen Variablen gesehen, um die Genese interregionaler Kooperation zu erklären. Er bezieht sich dabei auf eine realistische Konzeption von einem Wettbewerb zwischen verschiedenen Akteuren, die zu einer Akkumulation von Macht - auf individueller Basis oder als Teil einer temporären Koalition - führt. Institutionelle Macht und der Einfluss auf internationale Organisationen und globale ökonomische Prozesse sind zunehmend wichtige Ressourcen für Staaten, die versuchen, durch eine Hinwendung zu regionalen und in der Folge interregionalen Prozessen ihre Position im globalen System zu verbessern (Hulse 2016, S. 33). Die theoretische Interregionalismus-Debatte unterscheidet zwischen zwei Varianten von Balancing: dem (neo)realistisch geprägten „Power Balancing“ mit einer deutlichen militärischen Konnotation, und nichtmilitärischen Ausprägungen wie „Institutional Balancing“ (Valle 2008), „Soft Balancing“ (He und Feng 2008) oder „Hedging“ (Drechsel 2016), die Varianten eines Zusammenspiels realistischer und institutionalistischer Ansätze sind (Rüland 2014, S. 24). „Power balancing refers to the development of inter-regional links so as to restrict the abuse of power by third parties (can be one state or a group of nation-states). Accordingly, inter-regionalism is one approach for individual states to cooperate together and balance against a superior power or threatening alliance in the international realm" (Yi 2012, S. 50). Während das Thema Sicherheit, der zentrale Ankerpunkt des Balance-of-Power-Ansatzes, innerhalb der Triade unilateral oder in einigen Fällen intraregional verhandelt wird und es somit kaum Spielraum für interregionale Allianzen in diesem Bereich gibt, treten im Handlungsfeld der Wirtschaftsbeziehungen regelmäßig interregionale Bündnisse in Konkurrenz. Die globalen Wirtschaftsmächte in Europa, Asien und Nordamerika versuchen durch ein Institutional Balancing in interregionalen Foren ein wirtschaftliches Gleichgewicht aufrechtzuhalten, indem sie entweder untereinander oder mit Dritten in Kooperation treten. „The competitive dynamics between the powerful players leads them to seek out alliances with weaker regions in an attempt to tip the balance of economic power 
in their favour, or alternatively to exclude competitors from gaining a foothold in emerging economies" (Hulse 2016, S. 34). Folgt man dieser Logik des Institutional Balancing zwischen den großen Wirtschaftsblöcken und der Integration peripherer Wirtschaftsräume in interregionale Allianzen, lässt sich die Entstehung transregionaler Wirtschaftsräume als ein Wettlauf um wirtschaftliche Dominanz der jeweiligen Regionen interpretieren. (Hänggi et al. 2006; Rüland 2002a; Valle 2008). Die Aufnahme interregionaler Beziehungen ist in diesen Fällen ein Versuch eines Staates oder einer Gruppe von Staaten, ein Gegengewicht zu internationalen Konstellationen zu schaffen, die sie als Gefährdung ihrer (wirtschaftlichen) Interessen ansehen. Interregionale Prozesse bieten damit eine Antwort auf Verschiebungen im Machtgefüge der Triade und dienen zur Nivellierung externer regionaler Schemata, indem sie durch den Aufbau von Beziehungen zwischen zwei Regionen einen Ausgleich zu der Rolle einer dritten Region schaffen (Rüland 2002b, S. 2).

Laut Roloff (2001) und Rüland et al. (2008) lässt sich die Koinzidenz des intensiver werdenden wirtschaftlichen und institutionellen Wettbewerbs mit der gleichzeitigen Zunahme von Interdependenzen als ,concert of regions“ konzeptualisieren. „A , concert" may be a middle way between a balance of power system and cooperation albeit one that is closer to the paradigm of power than cooperation on a fictive continuum " (Rüland 2008; S. 18). Dabei können diese Beziehungen auch von Regionen außerhalb der Triade instrumentalisiert werden, um auf Veränderungen und Dynamiken im globalen System zu reagieren.

Institution Building beschreibt einerseits den Beitrag inter- und transregionaler Foren zum Prozess der Institutionalisierung der internationalen Beziehungen, andererseits den Effekt dieser Foren auf den institutionellen Ausbau intraregionaler Bündnisse. Die Etablierung interregionaler Strukturen und gemeinsamer Normen für die Interaktion mit regionsexternen Kooperationspartnern führt zu einer stärkeren Legitimation der Regionalen Organisation als außenpolitischer Akteur sowohl gegenüber den eigenen Mitgliedsstaaten als auch gegenüber Dritten. Strukturierte und institutionell gefestigte Dialoge können dabei eine größere Wirkmacht entfalten als reine Ad-hoc-Treffen zwischen interregionalen Akteuren. Aus Blickwinkel des Institutionalismus sind Institutionen und die damit verbundene Verriegelung des internationalen Systems zentraler Bestandteil von Kooperation. (Rüland 2002a, S. 7; Doidge 2011, S. 36). Institutionalisierte Dialogstrukturen bieten neben der rechtlichen Verstetigung interregionaler Prozesse auch einen Diskussionsrahmen für Fragestellungen, die bisher von den beteiligten Staaten oder Regionen nicht in anderen Foren diskutiert wurden (Doidge 2011, S. 36).

Interregionalismus bringt aber nicht nur einen Aufbau von Institutionen zwischen den beteiligten Regionalen Organisationen mit sich, sondern hat auch einen Effekt auf die interregionale Dialogstruktur. Der Bedarf an interner Konsensfindung und einheitlichen Positionen einer Region nimmt zu, wenn diese in einen interregionalen Dialog eintritt; somit steigt auch der Bedarf an institutioneller Ausgestaltung von Konsultationsmechanismen. „Intra-regionally, they [interregional Forums] become drivers of co-ordination as regional organizations seek to develop uniform positions in interregional dialogues. The putative result is more efficient information flows, more intensive intra-regional interaction and strengthened regional cohesion " (Rüland 2010, S. 1276). 
In der Literatur wird allerdings auch häufig die Fähigkeit von Interregionalismus, dem Aufbau von Institutionen dienlich zu sein, bezweifelt. Als größte Hindernisse werden dabei der geringe Grad von Institutionalisierung und die Vorliebe der beteiligten Partner für informelle, nicht bindende Entscheidungen benannt. (Muntschick 2012, S. 9; Pirozzi und Godsäter 2015).

Institutionalisierte Dialoge können auch als vorgeschalteter Diskussionsmechanismus für globale Angelegenheiten dienen. Sie üben dann ggf. eine RationalizerFunktion aus, indem sie Entscheidungsprozesse von einer multilateralen auf eine interregionale Ebene verlagern und so die Komplexität von Akteursstrukturen in globalen Foren verringern und eine Beschleunigung von Verhandlungsprozessen erreichen. „Viewing Interregionalism in terms of a rationalizing function predicts that deadlock at the multinational level will result in increased activity at the interregional level and that any agreements at the interregional level should be compatible with existing multilateral rules" (Hulse 2016, S. 35). Der Transfer von multinationalen Entscheidungsprozessen auf eine vorgelagerte Entscheidungsebene kann dabei helfen, verschiedene Probleme, die typischerweise mit internationalen Verhandlungen assoziiert werden, abzumildern. Sowohl die von Doidge (2011, S. 40) geschilderten Probleme von ,abdication and domination“, die durch die hervorgehobene Rolle einiger Staaten wie den USA, Russland oder China in internationalen Dialogen entstehen können, als auch ein drohender Stillstand der Verhandlungen durch die große Zahl divergenter Interessen in internationalen Foren sprechen für eine Rationalisierung des Entscheidungsprozesses auf regionaler und interregionaler Ebene. Eine ähnliche Rolle können interregionale Foren als Agenda Setter übernehmen, indem sie vorher vereinbarte Standpunkte in internationale Foren einbringen und damit ihre Position bei der Durchsetzung ihrer Interessen verbessern (Rüland 2002a, S. 10). Auch hinsichtlich der Agenda-Setter-Funktion argumentieren Autoren wie Doidge (2008a, 2011) oder Dent (2004, S. 228) mit den Vorteilen kleinerer Teilnehmerzahlen, größerer Kohäsion und gemeinsamer Interessen, die in interregionalen Foren vorherrschen. Durch die Vorabdiskussion von Entscheidungen, die auf globaler Ebene getroffen werden, können sich in interregionalen Foren bereits Koalitionen für oder gegen eine bestimmte Position bilden, die dann in den multilateralen Entscheidungsprozess transferiert werden (Doidge 2011, S. 45). Interregionale Foren können auch dazu dienen, Lösungen für existierende oder neu entstehende globale Probleme zu erörtern, und so Ausgangspunkte für eine breite globale Debatte bieten (Rüland 2010, S. 1277).

Als letzte der fünf Funktionen, die in den einschlägigen Studien interregionaler Beziehungen benannt werden, ist die von konstruktivistischen Überlegungen beeinflusste kollektive Identitätsbildung zu nennen (Gilson 2002, 2005; Ruggie 2002; Tsardanidis 2010). Dabei kann Identitätsbildung sowohl als eine Triebkraft für Interregionalismus wie auch lediglich als ein Ergebnis interregionaler Prozesse verstanden werden (Hulse 2014, S. 38). Betrachtet man die kollektive Identitätsbildung als möglichen Treiber von Interregionalismus, kann man sowohl eine interne Dimension, die sich auf die identitätsstiftende Wirkung der Wahrnehmung eigener Akteurskapazitäten bezieht, als auch eine externe Dimension, die Effekte dritter Akteure auf die Identität Regionaler Organisationen berücksichtigt, unterscheiden. Im Bereich der internen Identitätsbildung kann durch das Agieren einer Regiona- 
len Organisation als eigenständiger Akteur die Wahrnehmung der einzelnen Mitgliedsstaaten bezüglich einer regionalen Identität geschärft werden. Dieses Identity Building findet dann bereits auf regionaler und subregionaler Ebene statt und führt zu einer Identität jenseits nationaler Grenzen (Lucarelli und Fioramonti 2010, S. 4). Durch die Ausprägung einer eigenen Akteursqualität auf der außenpolitischen Bühne entwickelt die Region eine eigene Entität, die sie abgrenzbar gegenüber externen Gemeinschaften macht. Die Herausbildung institutionalisierter Dialogstrukturen mit anderen Regionen führt zu einer eigenen regionalen Wahrnehmung, die wiederum ein intrinsischer Bestandteil für die Herausbildung von Akteursqualitäten einer Region ist (Aggarwal and Fogarty 2004, S. 15). Diese Wechselwirkung zwischen Identität und internationaler Handlungsfähigkeit hat eine positive Auswirkung auf die Verdichtung interregionaler Dialoge. Regionale Akteure reflektieren ihre eigene Selbstwahrnehmung internationaler Präsenz auf mögliche Partner und regen damit Kooperation an (vgl. Gilson 2002; Grugel 2004). Neben der gesteigerten Selbstwahrnehmung der eigenen Handlungsfähigkeit als regionaler Akteur bietet der Kontakt mit externen Akteuren weitere Stimuli für die Herausbildung einer regionalen Identität (Doidge 2008a, S. 50). Dabei kann die Einbindung in interregionale Dialoge intendierte und nicht intendierte Effekte für die Ausprägung regionaler Identitäten haben und sowohl eine unmittelbare Konsequenz externer Einflüsse sein wie auch als eine reaktive Maßnahme auf dialogspezifische Kooperationsanreize erfolgen. In den Bereich der intendierten Einflussnahme auf regionale Identitätsbildung fällt sowohl die direkte Unterstützung regionaler Gruppierungen durch externe Akteure als auch die Artikulation einer Präferenz für regionale Dialogpartner dieser Akteure mit dem Ziel, integrative Prozesse in anderen Regionen einzuleiten. So versuchen etablierte Regionale Organisationen mithilfe interregionaler Dialoge, interne Kohärenz und die Übernahme regionaler Normen und Institutionen in Partnerregionen zu fördern (vgl. Hulse 2014, S. 39; Söderbaum 2005, S. 376; Stapel und Söderbaum 2017).

Eine Reihe von Fallstudien (Söderbaum und Stalgren 2010; Doidge 2011; Ribeiro-Hoffmann 2016, S. 604) hat daraufhin diese Funktionen auf verschiedene Arrangements wie ASEM oder EU-Mercosur angewendet und empirisch zu untermauern versucht. Andere Autoren kritisieren aber die theoretische Herleitung (Robles 2008) und den normativen Charakter (Camroux 2011, S. 201) der Funktionen und haben Anstrengungen unternommen, auf Grundlage weiterer theoretischer Modelle wie Putnams Zwei-Ebenen-Spiel neue Erklärungsansätze zu entwickeln, die jedoch in der neueren Interregionalismusforschung kaum rezipiert werden. Carmroux (2011 S. 212) bringt im Zusammenhang mit Interregionalismus die klassische Distinktion von Arnold Wolfers eines Milieu goals ins Spiel. Interregionalismus wird demnach von den Akteuren als eine Möglichkeit zur Umsetzung regionaler policies und Selbstprojektion im globalen Kontext genutzt, ohne den tatsächlichen Anspruch einer tiefgreifenden interregionalen Verdichtung anzustreben. Neben diesen Erklärungsversuchen, die sich auf die theoretischen Ansätze der IB berufen, gibt es auch alternative Erklärungsmodelle für die Entstehung und Auswirkung interregionaler Beziehungen, die sich außerhalb klassischer IB-Theorien verorten. Die Internationale Politische Ökonomie (IPÖ) sieht Interregionalismus als ein Bindeglied zwischen Staaten und dem globalen Markt, das vor allem durch die fehlende multilaterale Dynamik auf Ebene der WTO an Bedeutung gewonnen hat (McCrossan 2014, 
S. 36). Interregionale Arrangements zielen aus dieser stark ökonomisch orientierten Sichtweise vorrangig auf Handelsabkommen zwischen den beteiligten Regionen ab, während andere Faktoren für die Erklärung ihres Zustandekommens unberücksichtigt bleiben. Auch der Diffusionsansatz bietet eine Erklärung für die Entstehung interregionaler Arrangements (Börzel und Risse 2009; Jetschke und Lenz 2013). Aus dieser stark EU-zentrierten Sichtweise wird die Entwicklung solcher Arrangements in Verbindung mit der Diffusion des europäischen Regionalismusmodells in andere Weltregionen gebracht.

Während der hier eingeführte Funktionsbegriff einen Analyserahmen für die Untersuchung der Leistungsfähigkeit interregionaler Arrangements und der Interessen der beteiligten Akteure etabliert hat, ist dieser stark eurozentrisch geprägt und - wie im Folgenden erläutert wird - nur bedingt auf interregionale Arrangements jenseits der Triade übertragbar (Bersick 2008; Doidge 2011; Hardacre 2009). Die oben beschriebenen grundlegenden Funktionen von Interregionalismus sind ausschließlich von interregionalen Prozessen im Analysekontext der Außenbeziehungen der Europäischen Union abgeleitet und setzen die Beteiligung mindestens einer etablierten Regionalen Organisation mit ausgeprägter internationaler Handlungsfähigkeit voraus. Die EU nimmt aufgrund ihrer institutionellen und wirtschaftlichen Kapazitäten, ihrer normativen Integrationsdichte und internationalen Präsenz eine Sonderstellung unter der Vielzahl existierender ROs ein und Annahmen, die vor dem Hintergrund interregionaler Dialoge mit Beteiligung der EU getroffen wurden, lassen sich nicht ohne Weiteres auf andere Arrangements mit grundlegend anderer Akteurskonstellation übertragen (Dosch und Jacob 2010; Rüland 2014, S. 19). Funktionen, die sich aus dem Beziehungsgeflecht der EU mit anderen regionalen Partnern ableiten, müssen daher bei einem Transfer auf interregionale Kooperationen im Kontext einer Süd-Süd-Partnerschaft kritisch hinterfragt werden (Mattheis und Godsäter 2018).

In der aktuellen Interregionalismus-Literatur gibt es inzwischen einige Beispiele, die sich weniger auf die EU und das internationale System konzentrieren, sondern versuchen, die Effekte von Interregionalismus für die betreffenden außereuropäischen ROs abzubilden. So untersucht Plank (2017) die Auswirkungen der AU-EUKooperation auf den afrikanischen Sicherheitssektor und Doctor (2015) zeigt die integrativen Effekte von Interregionalismus für Mercosur. Auch Mattheis und Wunderlich (2017) versuchen in ihrer Studie zu Effekten von Interregionalismus auf die Akteurskapazitäten von Regionalen Organisationen die rein eurozentrische Perspektive $\mathrm{zu}$ verlassen und beziehen neben der EU auch ASEAN und Mercosur in ihre Untersuchung mit ein.

\section{Erweiterung des Funktionenbegriffes}

Globalisierung und Regionalisierung waren die bestimmenden Faktoren in der Restrukturierung des internationalen Systems nach Ende des Kalten Krieges. Diese beiden Prozesse treten besonders im Bereich des Wirtschaftssystems - das von seiner Struktur her durch ein geografisches Ungleichgewicht zugunsten von Nordamerika, Europa und Ostasien gekennzeichnet ist - komplementär auf (Holm und Sörensen 1995). Dieses Ungleichgewicht bei der Globalisierung wird von einer ebenso 
ungleichen Regionalisierung des wirtschaftlichen, politischen und sozialen Raumes begleitet. Zwar kam es nach Ende des Kalten Krieges zu einer neuen Welle regionalen Bewusstseins und einem weltweiten Anstieg der intraregionalen Kooperation, doch ist diese Entwicklung trotz ihrer globalen Dimension am stärksten in den Triade-Regionen und deren Peripherien zu beobachten, wo intraregionale Transaktionen seit den 1960er Jahren stark zugenommen haben (Hurrell 1995). Die Motivation, mit Regionen außerhalb der Triade zu kooperieren, korreliert stark mit der ökonomischen Bedeutung der jeweiligen Partnerregion. So ist Lateinamerika durch seine günstige geostrategische Lage und relative wirtschaftliche Bedeutung ein attraktives Ziel für transregionale Bemühungen seitens der Triade geworden, wohingegen Afrika sich bisher nicht als zugkräftiger Partner auf regionaler Ebene positionieren konnte und im interregionalen Netzwerk marginalisiert ist (Hänggi 2000; Söderbaum und Stalgren 2010). Auch die Interregionalismus-Forschung reflektiert auf einer institutionellen Ebene die Tatsache, dass Globalisierung ein ungleicher Prozess ist, der sich durch eine wachsende Verdichtung und Intensivierung der sozialen, politischen und wirtschaftlichen Verflechtungen innerhalb der OECD und einigen Schwellenländern abspielt, während Teile des globalen Südens kaum von positiven Globalisierungseffekten profitieren können.

Während die klassischen Funktionen von Interregionalismus einen Deutungsansatz für die Entstehung interregionaler Arrangements bieten, sieht Olivet (2005) dabei besonders die Balancing-Funktion, die für Beziehungen in der Triade eine herausragende Bedeutung einnimmt, als ungeeignet für die Erklärung peripherer interregionaler Arrangements. „Balancing power situations within the global structure of governance is the only one that actually addresses the reasons for the emergence of interregional relations within the Triad and the real interests behind it " (Olivet 2005, S. 12). Das Interesse der EU und der USA an trans- und interregionalen Foren erklärt sich danach vor allem durch die Möglichkeit, auf diesem Wege Herausforderungen innerhalb der vielschichtigen Global-Governance-Struktur zu begegnen. Auch für den Süd-Süd-Interregionalismus argumentiert van der Vleuten und Ribeiro Hoffmann (2013), dass die primäre Motivation der beteiligten ROs im Balancing des asymmetrischen Einflusses der EU und anderer einflussreicher westlicher Akteure liegt. Auch andere Autoren wie Mattheis und Wunderlich (2017) und Ribeiro-Hoffmann (2016) sehen gewisse Ansatzpunkte für eine globale Ausrichtung interregionaler Arrangements ohne Beteiligung der EU. So benennet etwa RibeiroHoffmann (2016, S. 607) Soft Balancing, als mögliche Erklärung für Süd-Süd-Interregionalismus. Allerdings zeigen Autoren wie He (2008, S. 365) und Rüland (2014) auf, dass auch Soft Balancing - das beispielsweise wirtschaftliche Sanktionen oder „strategic non-cooperation“ (Rüland 2014, S. 24) gegen Dritte umfasst - ein gewisses Maß an Akteurskapazitäten und wirtschaftlicher Einflussnahme voraussetzt, das in vielen Fällen die Möglichkeiten peripherer ROs übersteigt. Olivet (2005), Doidge (2011) und Mattheis und Godsäter (2018) weisen darauf hin das Balancing in seinen zahlreichen Facetten als Funktion von Interregionalismus nicht ohne Weiteres von der Triade auf Süd-Süd-Modelle übertragen werden kann. Zur Erklärung der Motivation peripherer Regionalorganisationen interregionale Kooperationen einzugehen werden daher zunehmend kapazitätsbildende Funktionen die nicht auf das globale System, sondern die Steigerung der eigenen Handlungsfähigkeit ausgerichtet sind, 
herangezogen. So finden sich inzwischen verschiedene empirische Fallbeispiele interregionaler Entwicklungskooperation - besonders im Kontext der EU-Außenbeziehungen - allerdings ist die theoretische Dimension dieser Beziehungen bisher kaum ausgeleuchtet (vgl. Söderbaum und Stalgren 2010; Mattheis und Godsäter 2018). Andere Autoren rücken hingegen die Interregionale Sicherheitszusammenarbeit in den Fokus. (Hanau-Santini et al. 2014; Plank 2017). Das Thema Sicherheit wurde mehr oder weniger prominent bei ASEM (Gilson 2005, S. 323; Hanau-Santini et al. 2014, S. 72), APEC, dem Regionalforum der ASEAN (ARF) - dort mit einem eigenen institutionalisierten sicherheitspolitischen Dialogforum - und im Rahmen der Partnerschaft zwischen AU und Europäischer Union (Pirozzi 2012) diskutiert.

Weder die Süd-Süd-Ausrichtung noch der asymmetrische Charakter von interregionalen Arrangements ist dabei eine Voraussetzung für die Analyse kapazitätsbildender Funktionen, und eine Vielzahl anderer Kooperationsformen kann einbezogen werden. Auch interregionale Arrangements zwischen dem europäischen Regionalismus und einer Regionalmacht des globalen Südens, die zumeist als Beleg für eine Institutionalisierung von Global Governance gesehen werden (Dent 2004; Vasconcelos 2008; Maihold 2010; Drechsel 2016), könnten durch einen Perspektivenwechsel als kapazitätsbildende Maßnahmen konzeptualisiert werden. Interregionalismus lässt sich daher immer als reaktive oder aktive Strategie von Regionen und den daran beteiligten Staaten begreifen und verfügt je nach Perspektive und Konstellation der Partner über eine eigenständige Funktionslogik.

Funding Open Access funding provided by Projekt DEAL.

Open Access Dieser Artikel wird unter der Creative Commons Namensnennung 4.0 International Lizenz veröffentlicht, welche die Nutzung, Vervielfältigung, Bearbeitung, Verbreitung und Wiedergabe in jeglichem Medium und Format erlaubt, sofern Sie den/die ursprünglichen Autor(en) und die Quelle ordnungsgemäß nennen, einen Link zur Creative Commons Lizenz beifügen und angeben, ob Änderungen vorgenommen wurden.

Die in diesem Artikel enthaltenen Bilder und sonstiges Drittmaterial unterliegen ebenfalls der genannten Creative Commons Lizenz, sofern sich aus der Abbildungslegende nichts anderes ergibt. Sofern das betreffende Material nicht unter der genannten Creative Commons Lizenz steht und die betreffende Handlung nicht nach gesetzlichen Vorschriften erlaubt ist, ist für die oben aufgeführten Weiterverwendungen des Materials die Einwilligung des jeweiligen Rechteinhabers einzuholen.

Weitere Details zur Lizenz entnehmen Sie bitte der Lizenzinformation auf http://creativecommons.org/ licenses/by/4.0/deed.de.

\section{Literatur}

Acharya, Amitav, und Alan Johnston. 2007. Crafting cooperation: the design and effect of regional institutions in comparative perspective. Cambridge: Cambridge University Press.

Aggarwal, Vinod 1998. Analyzing Institutional Transformation in the Asia-Pacific, In: Asia-Pacific crossroads. Regime creation and the future of APEC. Hrsg. V. Aggarwal, C.E. Morrison, 15-43 New York: St. Martin's Press

Aggarwal, Vinod, und Edward Fogarty. 2004. Between regionalism and globalism: European Union Transregional and inter-regional trade strategies. In European Union trade strategies: between globalism and regionalism, Hrsg. V. Aggarwal, E. Fogarty, 1-69. London: Palgrave.

Aggarwal, Vinod und Edward Fogarty. 2005. The Limits of Interregionalism: The EU and North America. Journal of European Integration 27(3):327-346 
Allison, Laura. 2015. The EU, ASEAN and Interregionalism Regionalism Support and Norm Diffusion between the EU and ASEAN. Basingstoke: Palgrave Macmillan.

Avery, William. 1973. The extra-regional transfer of integrative behavior. International Organization 27(4):549-556.

Baert, Francis, Tiziana Scaramagli, et al. 2014. Intersecting interregionalism: regions, global governance and the EU. United Nations University series on regionalism. Dordrecht: Springer.

Bersick, Sebastian. 2008. The democratization of inter- and transregional dialogues: the role of civil society, NGos and parliaments. In Asian-European relations: building blocks for global governance?, Hrsg. J. Rüland, G. Schubert, et al., 244-269. New York: Routledge.

Börzel, Tanja, und Thomas Risse. 2009. Diffusing (inter-) regionalism: the EU as a model of regional integration. KFG Working Paper Series, Nr. 7. Berlin: Freie Universität Berlin.

Bretherton, Charlotte, und Jürgen Vogler. 2006. The European Union as a global actor, 2. Aufl., London, New York: Routledge.

Cammack, Paul. 1999. Interpreting ASEM: Interregionalism and the new materialism. Journal of the Asia Pacific Economy 4(1):13-32.

Camroux, David. 2011. Interregionalism, a critique-the four levels of EU-ASEAN relations. In New regionalism and the European Union-dialogues, comparisons and new research directions, Hrsg. A. Warleigh-Lack, N. Robinson, et al., 201-217. New York: Routledge.

Carrapatoso, Astrid. 2011. Climate policy diffusion: interregional dialogue in China-EU relations. Global Change, Peace \& Security 23(2):177-194.

Costa, Olivier, und Clarissa Dri. 2014. How does the European parliament contribute to the construction of the EU's interregional dialogue. In Intersecting Interregionalism. United Nations University series on regionalism, Hrsg. F. Baert, T. Scaramagli, und F. Söderbaum, 129-150. Dordrecht: Springer.

Dent, Christopher. 2004. The Asia-Europe meeting and Interregionalism: toward a theory of multilateral utility. Asian Survey 44(2):213-236.

Doctor, Mahrukh. 2015. Interregionalism's impact on regional integration in developing countries: the case of Mercosur. Journal of European Public Policy 22(7):967-984.

Doidge, Matthew. 2008a. Regional organizations as actors in international relations: Interregionalism and asymmetric dialogues. In Asian-European relations: building blocks for global governance?, Hrsg. J. Rüland, G. Schuchert, et al., 32-54. New York: Routledge.

Doidge, Mathew. 2008b. Joined at the hip: regionalism and interregionalism. Journal of European Integration 29(2):229-248.

Doidge, Matthew. 2011. The European Union and Interregionalism: patterns of engagement. Farnham: Ashgate.

Dosch, Jörn, und Olaf Jacobs. 2010. Asia and Latin America-political, economic and multilateral relations. London: Routledge.

Drechsel, Benjamin. 2016. Interregionale Beziehungen und strategische Partnerschaften. Wiesbaden: Springer.

Engel, Ulf. 2018. Debatten Zur Epistemologie Und Periodisierung Von Regionalorganisationen. In Regionalismen, Hrsg. U. Engel, 18-25. Berlin: De Gruyter.

Farrell, Mary. 2004. The EU and Inter-Regional Cooperation: In Search of Global Presence?, UNU-CRIS e-working Papers W-2004/9. http://cris.unu.edu/sites/cris.unu.edu/files/W-2004-9.pdf. Zugegriffen: 30. Dez. 2019.

Faust, Jörg. 2004. Latin america, Chile and east asia: policy networks and successful diversification. Journal of Latin American Studies 36(4):747-770.

Faust, Jörg. 2006. Blueprint for an interregional future? The European Union and the southern cone. In $E U$ trade strategies between regionalism and globalism, Hrsg. V. Aggarwa, E. Fogarty, 41-63. Basingstoke: Palgrave McMillan.

Gardini, Gian Luca, und Andres Malamud. 2014. Interregionalism: concepts, types and critique-with a transatlantic focus. Atlantic Future Working Paper Nr. 38.

Gilson, Julie. 2002. Asia meets Europe: inter-regionalism and the Asia-Europe meeting. Cheltenham: Edward Elgar.

Gilson, Julie. 2005. New Interregionalism? The EU and East Asia. Journal of European Integration 27(3):307-326.

Grugel, Jean. 2004. New regionalism and modes of governance-comparing US and EU strategies in Latin America. European Journal of International Relations 10(4):603-626.

Hanau-Santini, Ruth, Sonja Lucarelli, et al. 2014. Interregionalism: a security study perspective. In Intersecting interregionalism regions, global governance and the EU, Hrsg. F. Baert, T. Scaramagli, et al., 71-88. Dordercht: Springer. 
Hänggi, Heiner, Ralf Roloff, et al. 2006. Interregionalism and international relations, a stepping stone to global governance? New York: Routledge.

Hänggi, Heiner. 2000. Interregionalism: empirical and theoretical perspectives., paper for the workshop "dollars, democracy and trade: external influence on economic integration in the americas" Los angeles, CA, May 18, 2000. http://www.cap.lmu.de/transatlantic/download/Haenggi.PDF. Zugegriffen: 30. Dez. 2019.

Hardacre, Allen. 2009. The rise and fall of Interregionalism in EU external relations. Dordrecht: Republic of Letters.

Hardacre, Alan, und Michael Smith. 2009. The EU and the diplomacy of complex interregionalism. The Hague Journal of Diplomacy 4(9):167-188.

He, Kai, und Huiyun Feng. 2008. If not soft balancing, then what? Reconsidering soft balancing and U.S. policy toward China. Security Studies 17(2):363-395.

Hettne, Björn. 2004. Interregionalism and world order. SGIR Fifth Pan-European International Relations Conference, Den Haag. Workingpaper.

Hettne, Björn. 2014. Regional actorship: a comparative approach to Interregionalism. In Intersecting Interregionalism United Nations University series on regionalism., Hrsg. F. Baert, T. Scaramagli, und F. Söderbaum, 55-70. Dordrecht: Springer.

Hettne, Björn. 2008. EU as a global actor: an anatomy of actorship, paper presented at the EU in international affairs conference 2008. http://www.ies.be/files/repo/conference2008/EUinIA_IV_1_Hettne.pdf. Zugegriffen: 30. Dez. 2019.

Holm, Hans Henrik, und Sørensen Georg. 1995. Whose world order? Uneven globalization and the end of the cold war. Boulder: Westview.

Hulse, Merran. 2014. Actorness beyond the European Union: comparing the international trade actorness of SADC and ECOWAS. Journal of Common Market Studies 52(3):547-565.

Hulse, Merran. 2016. United we stand, divided we fall: actorness and effectiveness of ECOWAS and SADC in interregional negotiations for economic partnership agreements. Dissertation. Nijmegen: Radboud University.

Hurrell, Andrew. 1995. Regionalism in theoretical perspective. In Regionalism in world politics, Hrsg. L. Fawcett, A. Hurrell, 37-73. Oxford: Oxford University Press.

Hwee, Yeo Lay, und Lluc López i Vidal. 2008. Regionalism and Interregionalism in the ASEM context: current dynamics and theoretical approaches. Documents Cibod, Asia Europe Foundation Nr. 23.

Jetschke, Anja, und Tobias Lenz. 2013. Does regionalism diffuse? A new research agenda for the study of regional organizations. Journal of European Policy 20(4):626-637.

Kaiser, Karl. 1968. The interaction of regional subsystems: some preliminary notes on recurrent patterns and the role of superpowers. World Politics 21(1):84-107.

Kaiser, Karl. 1981. Die Interaktion regionaler Subsysteme. In Regionalismus und regionale Integration. Zur Theorie der regionalen Integration, Hrsg. G. Doecker, F. Veitl, 99-121. Frankfurt: Peter Lang.

Keohane, Robert, und Joseph Nye. 1977. Power and interdependence. World politics in transition. Bosten: Little Brown.

Lucarelli, Sonia, und Lorenzo Fioramonti. 2010. External perceptions of the European Union as a global actor. London: Routledge.

Maihold, Günther. 2010. Conclusion: leadership coalitions as a new element for the EU's external action. In Europe and New Leading Powers: towards partnership in strategic policy areas, Hrsg. J. Husar, G. Maihold, et al., 149-156. Baden-Baden: Nomos.

Mattheis, Frank, und Andreas Godsäter. 2018. Interregionalism across the atlantic space. Wiesbaden: Springer VS.

Mattheis, Frank, und Uwe Wunderlich. 2017. Regional actorness and interregional relations: ASEAN, the EU and Mercosur. Journal of European Integration 39(6):723-738.

Maull, Hanns, und Nuria Okfen. 2003. Inter-regionalism in international relations: comparing APEC and ASEM. Asia Europe Journal 2003(1):237-249.

Maull, Hanns, und Nuria Okfen. 2006. Comparing interregionalism. The Asiapacificeconomic cooperation (APEC) and the Asia Europe meeting (ASEM). In Interregionalism and international relations, Hrsg. H. Hänggi, R. Roloff, et al., 217-233. London: Routledge.

McCrossan, Peter. 2014. The Political Economy of EU Interregionalism, Dissertation, Dublin City University. http://doras.dcu.ie/20296/1/PhD_Final_Thesis_October_2014.pdf. Zugegriffen: 30. Dez. 2019.

Muntschick, Johannes. 2012. Theorising regionalism and external influence: a situation structural approach. Mainz. Mainz Papers on International and European Politics, 2012/0.

Olivet, Marie Cecilia. 2005. Unravelling Interregionalism theory: a critical analysis of the new interregional relations between latin America and East Asia. Holanda: Transnational Institute. 
Panke, Diana. 2019. Regional cooperation through the lenses of states: Why do states nurture regional integration? The Review of International Organizations. https://doi.org/10.1007/s11558-019-09348$\mathrm{y}$.

Pirozzi, Nicoletta. 2012. Strengthening the africa-EU partnership on peace and security: how to engage African regional organizations and civil society. IAI Research Papers, Issue 6.

Pirozzi, Nicoletta, und Andreas Godsäter. 2015. The EU and Africa: regionalism and interregionalism beyond institutions. Atlantic Future Working Papers Nr. 26. Barcelona: Centre for International Affairs (CIDOB).

Plank, Friedrich. 2017. The effectiveness of interregional security cooperation: evaluating the joint engagement of the EU and the $\mathrm{AU}$ in response to the 2013 crisis in the central African republic. European Security 26(4):485-506.

Prieto, Germán. 2003. Constructing regionalism in South America: the role of ideas in the Andean Community and Mercosur projects. Colombian Economic Journal 1(1):267-303

Ravenhill, John. 2001. APEC and the construction of pacific rim regionalism. Cambridge: Cambridge University Press.

Ribeiro-Hoffmann, Andrea. 2016. Inter- and transregionalism. In The oxford handbook of comparative regionalism, Hrsg. T. Börzel, T. Risse, 600-618. Oxford: Oxford University Press.

Richards, Gareth, und Colin Kirkpatrick. 1999. Reorienting interregional co-operation in the global political economy: Europe's east Asian policy. Journal of Common Market Studies 37(4):683-710.

Robles, Alfredo 2008. The Asia-Europe Meeting: The Theory and Practice of Interregionalism. London: Routledge

Roloff, Ralf. 2001. Europe, America and Asia between globalization and regionalism: the interregional cooperation and the economic dimension of international politics. Paderborn: Ferdinand Schöningh.

Roloff, Ralf. 2006. Interregionalism in theoretical perspective: state of the art. In Interregionalism and International Relations, Hrsg. H. Hänggi, R. Roloff, et al., 17-31. New York: Routledge.

Ruggie, John Gerard. 2002. Constructing the world polity: essays on international Institutionalisation. New York: Routledge.

Rüland, Jürgen. 1999. The future of the ASEM process: who, how, why and what. In ASEM. The AsiaEurope meeting. A window of opportunity, Hrsg. W. Stokhof, P. van der Velde, 126-151. London: Kegan Paul International in association with International Institute for Asian Studies.

Rüland, Jürgen. 2001. ASEAN and the European Union: a bumpy interregional relationship. ZEI Discussion Paper, C 95. Bonn: Center for European Integration Studies, University of Bonn.

Rüland, Jürgen. 2002a. Trans- and Interregionalism: remarks on the state of the art of a new research Agenda, National Europe Center Paper Nr. 35. https://digitalcollections.anu.edu.au/bitstream/1885/ 41664/3/ruland2.pdf. Zugegriffen: 30. Dez. 2019.

Rüland, Jürgen. 2002b. ASEM and the emerging system of global governance. Paper presented at the Round Table: Asia-Europe Meeting (ASEM), Annual Meeting of the Association for Asian Studies, Washington, DC, 4-7 April 2002., 1-6.

Rüland, Jürgen. 2006. Interregionalism; an unfinished agenda. In Interregionalism and international relations, Hrsg. H. Hänggi, R. Roloff, et al., 295-313. New York: Routledge.

Rüland, Jürgen. 2008: Interregionalism and interregional cooperation: the case of Asia-Europe Relations. In Asian-European Relations: Building blocks for global governance? Hrsg. J. Rüland, G. Schubert, C. Storz, 3-31. London: Routledge

Rüland, Jürgen. 2010. Balancers, multilateral utilities or regional identity builders? International relations and the study of interregionalism. Journal of European Public Policy 17(8):1271-1283.

Rüland, Jürgen. 2014. Interregionalism and international relations: reanimating an obsolescent research agenda? In Intersecting Interregionalism regions, global governance and the EU, Hrsg. F. Baert, T. Scaramagli, et al., 15-35. Wiesbaden: Springer.

Rüland, Jürgen, Gunter Schuchert, et al. 2008. Asian-European relations: building blocks for global governance? New York: Routledge.

Santander, Sebastian. 2010. The ups and downs of interregionalism in latin America. In The EU and the global south, Hrsg. F. Söderbaum, P. Stalgren, 89-114. London: Lynne Rienner Publishers.

Söderbaum, Fredrik. 2005. The international political economy of regionalism. In Globalizing international political economy, Hrsg. N. Phillips, 221-245. Basingstoke: Palgrave.

Söderbaum, Frederik, und Patrik Stalgren. 2010. The EU and the global South. London: Lynne Rienner Publishers.

Stapel, Sören, und Frederik Söderbaum. 2017. Mapping and problematizing external funding to the AU and the RECs. 6-7 November 2017. GCGD conference paper. 
Tsardanidis, Charalambos. 2010. Inter-regionalism: a comparative analysis of ASEM and FEALAC. In Asia and Latin America political, economic and multilateral relations, Hrsg. J. Dosch, O. Jacob, 218-237. New York: Routledge.

Valle, Marina V. 2008. Interregionalism: a case study of the European Union and Mercosur. GARNET working paper Nr: 51/08.

de Vasconcelos, Álvaro. 2008. "Multilateralising" multipolarity. In Partnerships for effective multilateralism: EU relations with Brazil, China, India and Russia, Hrsg. G. Grevi, Á. de Vasconcelos, 11-32. Paris: The European Union Institute for Security Studies.

Väyrynen, Raimo. 2003. Regionalism: old and new. International Studies Review 5:25-51.

van der Vleuten, Anna, und Andrea Ribeiro Hoffmann. 2013. The politics of Interregionalism: relations between international regional organizations. In Routledge handbook of international organizations, Hrsg. B. Reinalda, 430-444. London: Routledge.

Waltz, Kenneth. 1979. Theory of international politics. New York: McGraw-Hill.

Wendt, Alexander. 1994. Collective identity formation and the international state. American Political Science Review 88(2):384-396.

Yamamoto, Yoshinobu. 1996. Regionalization in contemporary international relations. In Regionalization in the world economy: NAFTA, the Americas and Asia pacific, Hrsg. R. Van Whiting, 19-42. London: Macmillan.

Yi, Lai Suet. 2012. Inter-regionalism of nation-states: Asia-Europe Meeting (ASEM) as a case-study. Dissertation. Christchurch: University of Canterbury. 